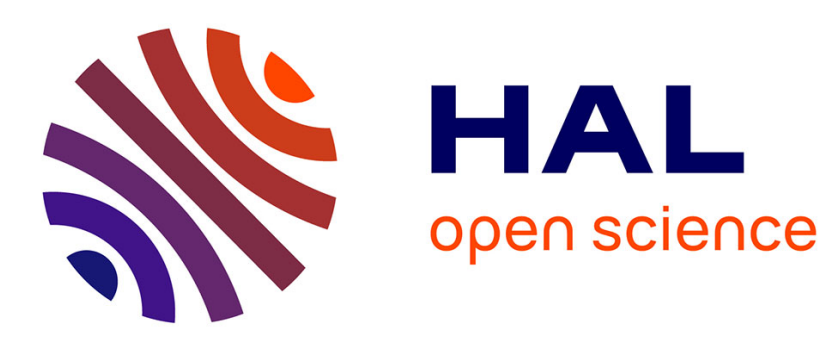

\title{
Global Synchronization Analysis of Droop-Controlled Microgrids: A Multivariable Cell Structure Approach
} Johannes Schiffer, Denis Efimov, Romeo Ortega

\section{To cite this version:}

Johannes Schiffer, Denis Efimov, Romeo Ortega. Global Synchronization Analysis of DroopControlled Microgrids: A Multivariable Cell Structure Approach. Automatica, 2019, 109, pp.108550. 10.1016/j.automatica.2019.108550 . hal-02177492v2

\section{HAL Id: hal-02177492 https://inria.hal.science/hal-02177492v2}

Submitted on 11 Jul 2019

HAL is a multi-disciplinary open access archive for the deposit and dissemination of scientific research documents, whether they are published or not. The documents may come from teaching and research institutions in France or abroad, or from public or private research centers.
L'archive ouverte pluridisciplinaire HAL, est destinée au dépôt et à la diffusion de documents scientifiques de niveau recherche, publiés ou non, émanant des établissements d'enseignement et de recherche français ou étrangers, des laboratoires publics ou privés. 


\title{
Global Synchronization Analysis of Droop-Controlled Microgrids - A Multivariable Cell Structure Approach
}

\author{
Johannes Schiffer $^{\mathrm{a}} \quad$ Denis Efimov $^{\mathrm{b}, \mathrm{c}} \quad$ Romeo Ortega $^{\mathrm{d}}$ \\ ${ }^{a}$ Brandenburgische Technische Universität Cottbus-Senftenberg, Siemens-Halske-Ring 14, 03046 Cottbus, Germany \\ ${ }^{\mathrm{b}}$ Inria, Univ. Lille, CNRS, UMR 9189 - CRIStAL, F-59000 Lille, France \\ ${ }^{\mathrm{c}}$ Department of Control Systems and Informatics, University ITMO, 197101 Saint Petersburg, Russia \\ ${ }^{\mathrm{d}}$ Laboratoire des Signaux et Systémes, École Supérieure d'Electricité (SUPELEC), Gif-sur-Yvette 91192, France
}

\begin{abstract}
The microgrid concept represents a promising approach to facilitate the large-scale integration of renewable energy sources. Motivated by this, the problem of global synchronization in droop-controlled microgrids with radial topology is considered. To this end, at first a necessary and sufficient condition for existence of equilibria is established in terms of the droop gains and the network parameters. Then, the local stability properties of the equilibria are characterized. Subsequently, sufficient conditions for almost global synchronization are derived by means of the multivariable cell structure approach recently proposed in [1]. The latter is an extension of the powerful cell structure principle developed by Leonov and Noldus to nonlinear systems that are periodic with respect to several state variables and possess multiple invariant solutions. The analysis is illustrated via numerical examples.
\end{abstract}

Key words: Microgrid stability, global synchronization, droop control, smart grid applications, complex networks

\section{Introduction}

\subsection{Motivation and existing literature}

The development of low-carbon, yet efficient and affordable, future energy systems requires a major transformation of today's power systems [2,3]. In particular, the increasing penetration of volatile distributed generation (DG) units on the low (LV) and medium voltage (MV) levels calls for new power system operation paradigms. The microgrid (MG) has been identified as a fundamental concept to facilitate this transition [4-6].

A MG is an electrically connected subsystem of a larger power system, which is connected to the main system via a single point of connection termed point of common

\footnotetext{
^ Corresponding author J. Schiffer. Tel. +49-355-692809. Fax +49-355-692453.

Email addresses: schiffer@b-tu.de (Johannes Schiffer), Denis.Efimov@inria.fr (Denis Efimov), ortega@lss.supelec.fr (Romeo Ortega).
}

coupling (PCC) [4,5,7]. A typical MG is comprised of several DG units, loads and, possibly, storage devices. Compared to conventional distribution networks, MGs have the capability of operating either in grid-connected mode or islanded mode, i.e., completely isolated from the main system. Therefore they have, among other benefits, the potential to significantly enhance network resilience [6]. Clearly, to fully exploit the new possibilities, which can be offered by MGs, adequate control strategies are needed $[5,8]$. This necessity is further evidenced by the fact that most DG units are interfaced to the network via power inverters, instead of synchronous generators (SGs) as in conventional power systems $[9,10,7]$.

In the present paper, we focus on frequency synchronization, which - as in any alternating current (AC) power system - is a prime control objective in MGs [5,8]. As such it has received significant attention in recent years and there are a large variety of possible control schemes to address this objective, such as droop control [11,10], virtual synchronous machines [12-14], virtual oscillator control [15] and frequency control schemes implemented 
in a synchronous $d q$ reference frame [16-19].

As of today, the most commonly employed control scheme in MGs is droop control $[5,10,6]$. Droop control is a decentralized proportional control scheme, which can jointly achieve frequency synchronization and power sharing $[5,10,6]$. The latter is an important additional control objective in MGs, since it allows to specify the steady-state utilization of the DG units relative to each other, while at the same time satisfying the load demand in the MG [20]. Furthermore, droop control has the advantage of allowing for a seamless joint operation of inverter- and SG-interfaced units [21] and being able to provide robustness with respect to time delays $[22,23]$ as well as clock drifts [24].

Compared to these features, the satisfactory performance of the control schemes derived in [16-18] critically relies on the assumption that the internal clocks of the DG units are synchronized by a global time synchronization signal provided through a global positioning or communication system [10]. Furthermore, the approaches in [15-18] can not ensure a desired power sharing.

A decentralized control scheme for inverters ensuring almost global convergence of trajectories has recently been proposed in $[25,26]$. Yet, the approach is restricted to purely inverter-based networks and the convergence result requires the complete a-priori knowledge of the stationary solution, which is difficult to obtain in practice.

\subsection{Contributions}

The main contribution of the paper is to provide a global synchronization analysis of droop-controlled MGs with radial topology. The latter is a frequently encountered feature in MGs [10] and distribution networks [27]. Furthermore, the benchmark models developed by the CIGRE Task Force C6.04.02 indicate that a radial topology will also be relevant in future distribution networks $[28,29]$. For that purpose and since the synchronization frequency is identical for all DG units we perform a change of coordinates, which allows to cast the (global) synchronization problem as a (global) stability analysis problem. In contrast to the present work, all available analytic stability conditions for droop-controlled MGs, e.g., [20,30,31], permit to assert only local stability properties.

The MG dynamics and its stationary equations, as those of any AC power system, are nonlinear and periodic with respect to certain state variables (namely, the phase angles). As a consequence, the MG possesses multiple invariant solutions. In the context of stability investigation for nonlinear systems with multiple invariant sets, the Lyapunov function method is the main tool, which provides necessary and sufficient stability conditions [32$40]$. Yet, the main difficulty frequently encountered in the application of Lyapunov methods to practical systems is the lack of constructive techniques for the design of suitable Lyapunov functions. Therefore, any extension of this methodology geared towards relaxing the usual Lyapunov stability requirements is of great importance in applications.

For the present case of a system with periodic dynamics, Leonov [41,42] and Noldus [43] proposed the cell structure approach, which significantly relaxes the positive definiteness and smoothness requirements of the standard Lyapunov function approach, while allowing to assess Lagrange stability of the system. These properties have recently been exploited to provide global stability analyses for power systems with conventional synchronous generators $[44,45]$. Yet, these analyses are restricted to the single-machine-infinite-bus scenario, because the cell structure approach of Leonov and Noldus is only applicable to systems, whose dynamics are periodic with respect to a scalar state variable. This fundamental drawback has motivated the development of a multivariable cell structure framework and the concept of a Leonov function in $[46,1]$.

Unlike the original cell structure framework, the approach in $[46,1]$ is applicable to nonlinear systems, whose dynamics are periodic with respect to several state variables and which possess multiple invariant solutions. Clearly, both latter properties are inherent features of MGs. Furthermore, compared to standard Lyapunov theory $[47,48]$, the use of Leonov functions permits to relax the usual sign definiteness requirements on the Lyapunov function and its time-derivative by exploiting the periodicity of the system dynamics. This relaxation is essential to provide conditions for global boundedness of trajectories in the MG case.

In summary, the presented analysis consists of the following four main items:

- Derive necessary and sufficient conditions for existence of synchronized solutions and their local stability properties.

- Provide sufficient conditions for global boundedness of trajectories via the multivariable cell structure approach recently proposed in $[46,1]$.

- By using these results, establish almost global asymptotic stability of the desired equilibrium set of the MG, i.e., we show that for all initial conditions, except a set of measure zero, the solutions of the MG converge to an asymptotically stable equilibrium point.

- Illustrate the efficiency of the proposed conditions numerically via a MG based on the CIGRE benchmark MV distribution network [29]. To this end, we also suggest a numerical evaluation procedure.

A discussion on the physical implications of the derived conditions is also provided in the paper. We believe 
that - even though being based on a simplified modelthe present analysis contributes towards a more complete understanding of the synchronization problem in MGs by providing a very different (global) perspective on the problem compared to the available literature. In addition, our results are directly applicable to the synchronization problem of second-order Kuramoto oscillators $[49,50]$ and the employed approach has also the potential to be used - with appropriate modificationsin the global analysis of more generic complex oscillator networks [51].

Furthermore, the analysis in the present paper extends our related work [52] in several respects: first, we present necessary and sufficient conditions for existence of equilibria and their local stability properties; second, we provide a detailed proof of our main result; third, we discuss the (physical) implications of the proposed stability conditions; finally, we suggest a numerical evaluation procedure to verify our conditions.

The remainder of the paper is structured as follows. The employed MG model is introduced in Section 2 and its equilibrium set is characterized. The conditions for almost global synchronization are given in Section 3. Numerical examples to illustrate the derived conditions are presented in Section 4. Conclusions and an outlook on future work are given in Section 5 .

Notation. We define the sets $\mathbb{R}_{\geq 0}:=\{x \in \mathbb{R} \mid x \geq 0\}$, $\mathbb{R}_{>0}:=\{x \in \mathbb{R} \mid x>0\}$ and $\mathbb{S}:=[0,2 \pi)$. The set of complex numbers is denoted by $\mathbb{C}$ and that of nonnegative integers by $\mathbb{Z}_{>0}$. For a set $\mathcal{V},|\mathcal{V}|$ denotes its cardinality. For a set of, possibly unordered, positive natural numbers $\mathcal{V}=\{l, k, \ldots, N\}$, the short-hand $i \sim$ $\mathcal{V}$ denotes $i=l, k, \ldots, N$. Given a positive integer $N$, $\underline{0}_{N} \in \mathbb{R}^{N}$ denotes the vector of all zeros, $\mathbb{1}_{N} \in \mathbb{R}^{N}$ the vector with all ones and $\mathbf{I}_{N}$ the $N \times N$ identity matrix. Let $x=\operatorname{col}\left(x_{1}, \ldots, x_{N}\right) \in \mathbb{R}^{N}$ denote a column vector with entries $x_{i} \in \mathbb{R}$. Whenever clear from the context, we simply write $x=\operatorname{col}\left(x_{i}\right) \in \mathbb{R}^{N}$. Let $\operatorname{diag}\left(a_{i}\right) \in \mathbb{R}^{N \times N}$ denote a diagonal matrix with entries $a_{i} \in \mathbb{R}$. Let $j$ denote the imaginary unit. The complex conjugate of a vector $v \in \mathbb{C}^{N}$ is denoted by $v^{*}$. For a matrix $A \in \mathbb{R}^{N \times N}, \lambda_{\max }(A)$, respectively $\lambda_{\min }(A)$, denotes the maximum, respectively minimum, eigenvalue of $A$. For a (smooth) function $f: \mathbb{R}^{N} \rightarrow \mathbb{R}, \nabla f$ denotes its (transposed) gradient and $\nabla^{2} f$ its Hessian. We employ the short-hands $\nabla f\left(x^{*}\right):=\left.\nabla f(x)\right|_{x=x^{*}}$ and $\nabla^{2} f\left(x^{*}\right):=\left.\nabla^{2} f(x)\right|_{x=x^{*}}$. Furthermore, $|x|=\sqrt{x^{\top} x}$ denotes the usual Euclidean norm of a vector $x \in \mathbb{R}^{N}$ and $|x|_{\infty}=\max _{i}\left|x_{i}\right|$ its infinity norm. For $x \in \mathbb{R}^{N}$, the element-wise sine, cosine, arcsine and tanh functions are denoted by $\sin (x) \in[-1,1]^{N}, \cos (x) \in[-1,1]^{N}$, $\arcsin (x) \in\left[-\frac{\pi}{2}, \frac{\pi}{2}\right]^{N}$ (with $\|x\|_{\infty} \leq 1$ ) and $\tanh (x) \in$ $[-1,1]^{N}$, respectively. Also, $\tanh ^{2}(x)$ denotes the square function applied element-wise to $\tanh (x)$.

\section{Microgrid model, synchronized motions and equilibria}

\subsection{Microgrid model}

We consider a MG with mixed rotational and inverterinterfaced generation pool [21,7]. The topology of the electrical network is described by an undirected and connected graph $\mathcal{G}=(\mathcal{N}, \mathcal{E})$, where the set of network nodes is denoted by $\mathcal{N}=\{1,2, \ldots, N\}, N>1$ and the set of edges (representing power lines) by $\mathcal{E}=\left\{e_{1}, \ldots, e_{m}\right\}$, see [7]. Furthermore, by associating an arbitrary ordering to the edges, we introduce the node-edge incidence matrix $\mathcal{B} \in \mathbb{R}^{N \times m}$, the entries of which are defined as $b_{i l}=1$ if node $i$ is the source of the $l$-th edge $e_{l}, b_{i l}=-1$ if $i$ is the sink of $e_{l}$ and $b_{i l}=0$ otherwise.

We associate a phase angle $\theta_{i}: \mathbb{R}_{\geq 0} \rightarrow \mathbb{R}$ and a corresponding electrical frequency $\omega_{i}=\dot{\theta}_{i}$ to each node $i \in \mathcal{N}$. In contrast to their more usual definition in $\mathbb{S}^{N}$ (i.e., the $N$-dimensional torus), it is essential for the subsequent analysis to define the angles in $\mathbb{R}^{N}$. This permits us to construct a continuous Lyapunov-like function (see Section 3.4), which contains linear terms in the angles $\theta_{i}$ and is instrumental to establish our main global stability claim ${ }^{1}$. Following the usual approach in frequency synchronization studies [55,30], we assume that the voltage amplitudes at all nodes are positive real constants. Furthermore, we assume that all line impedances are purely inductive. This can be justified as follows [30,20]. Although in MV and LV networks the line impedance is typically not solely inductive, both the output (LCL) filter and the output transformer impedances of a DG unit are usually mainly inductive. In this case, the resistive part of the admittances are dominated by the inductive ones. This applies in particular to MGs on the MV level. We only consider such MGs and absorb the inverter output admittance into the line admittances, while neglecting all resistive effects. In the present case this assumption is additionally justified since the considered droop control of [11] is mainly used in MGs with dominantly inductive admittances [10]. Hence, if node $i \in \mathcal{N}$ is connected to node $k \in \mathcal{N}$, this is represented by a nonzero line susceptance $B_{i k} \in \mathbb{R}_{<0}$. If these nodes are not connected via a power line, then $B_{i k}=0$. The set of neighbors of a node $i \in \mathcal{N}$ is denoted by $\mathcal{N}_{i}:=\left\{k \mid k \in \mathcal{N}, k \neq i, B_{i k} \neq 0\right\}$. Under the made assumptions, the active power flow $P_{i}: \mathbb{R}^{\left|\mathcal{N}_{i}\right|} \rightarrow \mathbb{R}$ at node $i$ is given by ${ }^{2}$ [55]

$$
P_{i}=G_{i i} V_{i}^{2}+\sum_{k \sim \mathcal{N}_{i}}\left|B_{i k}\right| V_{i} V_{k} \sin \left(\theta_{i k}\right),
$$

\footnotetext{
1 Defining the phase angles in Euclidean space is also a common step in local Lyapunov-based stability analysis of power systems and MGs [53,20,54,31].

2 To simplify notation, the time argument of all signals is omitted in the sequel.
} 
where we have introduced the standard short-hand notation $\theta_{i k}=\theta_{i}-\theta_{k}$ and $V_{i} \in \mathbb{R}_{>0}$ and $V_{k} \in \mathbb{R}_{>0}$ denote the voltage amplitudes at the $i$-th, respectively $k$-th, node. Moreover, $G_{i i} \in \mathbb{R}_{>0}$ represents the shunt conductance, i.e., the active power load ${ }^{3}$, at node $i$.

Furthermore, we assume that all generation units are equipped with the standard droop control $[55,10]$. Then, as shown in $[21,20]$, the dynamics of both rotational and inverter-interfaced generators can be modeled by the well-known swing equation [55]. Thus the dynamics of the unit at the $i$-th node, $i \in \mathcal{N}$, are given by [21,20]

$$
\begin{aligned}
\dot{\theta}_{i} & =\omega_{i}, \\
M_{i} \dot{\omega}_{i} & =-\frac{1}{k_{i}}\left(\omega_{i}-\omega^{d}\right)-P_{i}+P_{i}^{d},
\end{aligned}
$$

where $k_{i} \in \mathbb{R}_{>0}$ is the frequency droop gain, and thus a design parameter, $\omega^{d} \in \mathbb{R}_{>0}$ is the nominal electrical frequency, $P_{i}^{d} \in \mathbb{R}$ is the active power setpoint and the active power flow $P_{i}$ is given by (1). For rotationally interfaced units, $M_{i} \in \mathbb{R}_{>0}$ denotes the inertia constant of the machine, while for inverter-interfaced units the virtual inertia constant is given by $M_{i}=\tau_{P_{i}} / k_{i}$, where $\tau_{P_{i}} \in \mathbb{R}_{>0}$ is the time constant of the power measurement filter [20,7]. Thus, in general, for inverter-interfaced units $M_{i}$ is also a design parameter. However, to simplify the presentation, in the sequel we assume $M_{i}$ is constant for both rotational and inverter-interfaced units. This is justified by the fact that for any given $M_{i}$ and $k_{i}$, we can select a corresponding $\tau_{P_{i}}$. For further details on the employed modeling procedure, we refer the reader to [7].

For our subsequent analysis, we make the following assumption on the MG topology, which is commonly encountered in distribution networks [10,27-29].

Assumption 1 The graph $\mathcal{G}$ describing the topology of the system $(2), i=1, \ldots, N$, is a tree.

With Assumption 1, $\mathcal{B}$ has full column rank, i.e., $m=$ $N-1$. Since then $\mathcal{B}$ has linearly independent columns, its pseudo-inverse $\mathcal{B}^{+}$is given by

$$
\mathcal{B}^{+}=\left(\mathcal{B}^{\top} \mathcal{B}\right)^{-1} \mathcal{B}^{\top}
$$

and $\mathcal{B}^{+}$is a left-inverse of $\mathcal{B}$, i.e., $\mathcal{B}^{+} \mathcal{B}=\mathbf{I}_{(N-1)}$.

In order to write the system $(1),(2), i \sim \mathcal{N}$, compactly, we introduce the matrices

$$
M=\operatorname{diag}\left(M_{i}\right) \in \mathbb{R}_{>0}^{N \times N}, K=\operatorname{diag}\left(k_{i}\right) \in \mathbb{R}_{>0}^{N \times N},
$$

\footnotetext{
3 For constant voltage amplitudes, any constant power load can equivalently be represented by a constant impedance load, i.e., to any constant $P \in \mathbb{R}_{>0}$ and constant $V \in \mathbb{R}_{>0}$, there exists a constant $G \in \mathbb{R}_{>0}$, such that $P=G V^{2}$. Similarly, a constant current load $I \in \mathbb{R}_{>0}$ can be represented by $I=G V$.
}

and the vectors

$$
\begin{aligned}
\theta & =\operatorname{col}\left(\theta_{i}\right) \in \mathbb{R}^{N}, \omega=\operatorname{col}\left(\omega_{i}\right) \in \mathbb{R}^{N}, \\
P^{\text {net }} & =\operatorname{col}\left(P_{i}^{d}-G_{i i} V_{i}^{2}\right) \in \mathbb{R}^{N} .
\end{aligned}
$$

With $a_{m}=V_{i} V_{k}\left|B_{i k}\right|, m=1, \ldots, N-1$, we define the diagonal matrix of line weights

$$
A=\operatorname{diag}\left(a_{m}\right) \in \mathbb{R}^{(N-1) \times(N-1)},
$$

and the potential function $U: \mathbb{R}^{N} \rightarrow \mathbb{R}$,

$$
\begin{aligned}
U(\theta) & =-\sum_{\{i, k\} \in \mathcal{N} \times \mathcal{N}} V_{i} V_{k}\left|B_{i k}\right| \cos \left(\theta_{i k}\right) \\
& =-\mathbb{1}_{(N-1)}^{\top} A \cos \left(\mathcal{B}^{\top} \theta\right) .
\end{aligned}
$$

The potential $U(\theta)$ and its gradient,

$$
\nabla U(\theta)=\mathcal{B} A \sin \left(\mathcal{B}^{\top} \theta\right)
$$

possess the following symmetry properties for any $\alpha \in \mathbb{R}$,

$$
U\left(\theta+\alpha \mathbb{1}_{N}\right)=U(\theta), \quad \nabla U\left(\theta+\alpha \mathbb{1}_{N}\right)=\nabla U(\theta) .
$$

These symmetry properties arise from the fact that the power flows (1) only depend upon angle differences. Furthermore, since $\mathbb{1}_{N}^{\top} \mathcal{B}=\underline{0}_{N}$,

$$
\mathbb{1}_{N}^{\top} \nabla U(\theta)=0
$$

Then, the system (1), (2), $i \sim \mathcal{N}$, can be written compactly as

$$
\begin{aligned}
\dot{\theta} & =\omega, \\
M \dot{\omega} & =-K^{-1}\left(\omega-\omega^{d} \mathbb{1}_{N}\right)-\nabla U(\theta)+P^{\text {net }} .
\end{aligned}
$$

Remark 2 In addition to a MG, the model (9) can also represent a bulk power system, as well as a second-order Kuramoto model. For $N=1$ the model (9) reduces to a nonlinear pendulum, see [56, Section V].

\subsection{Synchronized motions and equilibria}

We employ the definition below to characterize desired invariant solutions of the system (9).

Definition 3 The system (9) admits a synchronized motion if it has an invariant solution for all $t \geq 0$ of the form

$$
\theta^{s}(t)=\omega^{s} t+\theta_{0}^{s}, \quad \omega^{s}=\omega^{*} \mathbb{1}_{N},
$$

where $\omega^{*} \in \mathbb{R}$ and $\theta_{0}^{s} \in \mathbb{R}^{n}$ such that

$$
\left|\theta_{0, i}^{s}-\theta_{0, k}^{s}\right|<\frac{\pi}{2} \quad \forall i \in \mathcal{N}, \forall k \in \mathcal{N}_{i}
$$


Note that the shift-invariance properties (7) have the following well-known implication for synchronized motions of the system (9). If the system (9) possesses a synchronized motion $\theta^{s}(t)=\omega^{s} t+\theta_{0}^{s}+\alpha \mathbb{1}_{N}, \omega^{s}=\omega^{*} \mathbb{1}_{N}$ with $\alpha=0$, then it always admits an infinite number of synchronized solutions for $\alpha \in \mathbb{R}$. In addition, from (8) and (9), it follows that for $\dot{\omega}^{s}=\underline{0}_{N}[20]$

$$
\mathbb{1}_{N}^{\top} M \dot{\omega}^{s}=0 \Rightarrow \omega^{*}=\omega^{d}+\frac{\mathbb{1}_{N}^{\top} P^{\text {net }}}{\mathbb{1}_{N}^{\top} K^{-1} \mathbb{1}_{N}}
$$

Therefore, $\omega^{*}$ is uniquely defined by $P^{\text {net }}$ and $K$.

Motivated by these observations, we eliminate the invariant subspace of solutions of (9) through an appropriate coordinate transformation. Since we focus our analysis on tree networks and, inspired by $[57,31,58]$, we introduce the new variable

$$
\eta=\mathcal{B}^{\top} \theta \in \mathbb{R}^{(N-1)},
$$

where we recall that $\mathcal{B}$ is the network incidence matrix. Thus, $\eta$ defines the phase angle differences between the nodes and is a projection of $\theta$ on the subspace orthogonal to $\mathbb{1}_{N}$, i.e., for any constant $\theta_{\mathrm{av}} \in \mathbb{R}$,

$$
\eta=\mathcal{B}^{\top}\left(\theta+\theta_{\mathrm{av}} \mathbb{1}_{N}\right)=\mathcal{B}^{\top} \theta
$$

Furthermore, with the change of variables (11) it also follows from (5) that

$$
\begin{aligned}
U(\eta) & =-\mathbb{1}_{(N-1)}^{\top} A \cos (\eta), \\
\nabla U(\eta) & =A \sin (\eta), \quad \nabla U^{2}(\eta)=A \cos (\eta),
\end{aligned}
$$

and thus from (6) that

$$
\nabla U(\theta)=\mathcal{B} A \sin \left(\mathcal{B}^{\top} \theta\right)=\mathcal{B} \nabla U(\eta)
$$

Consequently, in reduced coordinates, the dynamics (9) are given by

$$
\begin{aligned}
\dot{\eta} & =\mathcal{B}^{\top} \omega \\
M \dot{\omega} & =-K^{-1}\left(\omega-\omega^{d} \mathbb{1}_{N}\right)-\mathcal{B} \nabla U(\eta)+P^{\text {net }} .
\end{aligned}
$$

Clearly, appearance of a synchronized motion of the system (9) corresponds to an equilibrium of (13), i.e.,

$\eta^{*}=\mathcal{B}^{\top}\left(\theta_{0}^{s}+\omega^{s} t+\alpha \mathbb{1}_{N}\right)=\mathcal{B}^{\top}\left(\theta_{0}^{s}+\left(\omega^{*} t+\alpha\right) \mathbb{1}_{N}\right)=\mathcal{B}^{\top} \theta_{0}^{s}$.

Furthermore, asymptotic stability of $\operatorname{col}\left(\eta^{*}, \omega^{s}\right)$ is equivalent to asymptotic convergence of the solutions $\operatorname{col}(\theta, \omega)$ to $\operatorname{col}\left(\theta^{s}, \omega^{s}\right)$ - up to a constant uniform shift $\theta_{\mathrm{av}} \mathbb{1}_{N}$, $\theta_{\mathrm{av}} \in \mathbb{R}$, in all angles.

\subsection{A necessary and sufficient condition for existence of equilibria}

We provide a necessary and sufficient condition for existence of equilibria of the system (13). The condition is similar to that derived for first-order MG systems in [30, Theorem 2]. But compared to the implicit characterization of solutions based on the edge flows and the pseudo-inverse of the network Laplacian in [30, Theorem 2], we provide an explicit characterization of the solutions in terms of the system parameters by using the pseudo-inverse $\mathcal{B}^{+}$of the network incidence matrix. For first-order Kuramoto oscillator dynamics, the number of equilibria has also been identified previously in $[59$, Corollary 2], but therein the equilibria are not explicitly given.

Proposition 4 Consider the system (13) with Assumption 1. Recall the matrices $\mathcal{B}^{+}$in (3) and $A$ in (4). The system (13) possesses equilibria if and only if

$$
\left\|A^{-1} \mathcal{B}^{+}\left(P^{\text {net }}-\frac{\mathbb{1}_{N}^{\top} P^{\text {net }}}{\mathbb{1}_{N}^{\top} K^{-1} \mathbb{1}_{N}} K^{-1} \mathbb{1}_{N}\right)\right\|_{\infty} \leq 1 .
$$

Then, also all equilibria are isolated. Furthermore, if and only if (15) is satisfied with strict inequality, then the equilibria are given by $\operatorname{col}\left(\eta_{0}^{s, i}, \omega^{*} \mathbb{1}_{N}\right)$ (modulo $\left.2 \pi\right)$, where $\eta_{0}^{s, i}, i=1, \ldots, 2^{(N-1)}$, are permutations of the vectors $\eta_{0}^{s}$ and

$$
\hat{\eta}_{0}^{s}=\pi \mathbb{1}_{(N-1)}-\eta_{0}^{s},
$$

with

$\eta_{0}^{s}=\arcsin \left(A^{-1} \mathcal{B}^{+}\left(P^{\text {net }}-\frac{\mathbb{1}_{N}^{\top} P^{\text {net }}}{\mathbb{1}_{N}^{\top} K^{-1} \mathbb{1}_{N}} K^{-1} \mathbb{1}_{N}\right)\right)$.

PROOF. An equilibrium of (13) has to satisfy

$$
P^{\text {net }}-\left(\omega^{*}-\omega^{d}\right) K^{-1} \mathbb{1}_{N}=\mathcal{B} \nabla U\left(\eta_{0}^{s}\right),
$$

with $\omega^{*}$ uniquely given by (10). With Assumption 1 , by left-multiplying with $\mathcal{B}^{+}$, see $(3)$, we obtain

$$
\mathcal{B}^{+}\left(P^{\text {net }}-\left(\omega^{*}-\omega^{d}\right) K^{-1} \mathbb{1}_{N}\right)=\nabla U\left(\eta_{0}^{s}\right)=A \sin \left(\eta_{0}^{s}\right) .
$$

Since $A>0$ and replacing $\omega^{*}$ with (10), the above is equivalent to

$$
A^{-1} \mathcal{B}^{+}\left(P^{\text {net }}-\frac{\mathbb{1}_{N}^{\top} P^{\text {net }}}{\mathbb{1}_{N}^{\top} K^{-1} \mathbb{1}_{N}} K^{-1} \mathbb{1}_{N}\right)=\sin \left(\eta_{0}^{s}\right),
$$

which is a system of $N-1$ decoupled nonlinear equations that has solutions if and only if (15) is satisfied. Clearly, each of these equations has at most two distinct 
solutions given by (17), and exactly two solutions given by (16) if and only if (15) is satisfied with strict inequality, completing the proof.

Remark 5 Condition (15) has the following physical interpretation. The stationary active power flow injections are given by (18). For a network with radial topology, by left-multiplication with $\mathcal{B}^{+}$these active power injections can be expressed in terms of the stationary power flows across the individual power lines of the microgrid model (13). Hence, condition (15) states that for existence of equilibria of the system (13) it is necessary and sufficient that the magnitudes of the stationary active power flows along the individual power lines do not exceed the maximal possible power flows, i.e., $\max _{\eta_{m}^{s} \in \mathbb{R}}\left(a_{m} \sin \left(\eta_{m}^{s}\right)\right)=a_{m}, m=1, \ldots, N-1$, see $(4)$. This interpretation is also in line with the observations of [30, Remark 3].

\section{Almost global synchronization of droop- controlled MGs}

This section is dedicated to the analysis of global synchronization in radial droop-controlled MGs. Our analysis is based on the recently proposed multivariable cell structure approach [46,1] and relies on constructing a suitable Leonov function for the MG system. To this end, we perform the following three steps. First, we establish local stability properties of all equilibria of the system (13) and construct a suitable error system in Section 3.1. Then, in Section 3.3 we establish a sufficient condition for boundedness of trajectories by using the multivariable cell structure approach and the concept of a Leonov function, which were recently introduced in [46,1]. This is a fundamental prerequisite for the main global synchronization analysis - the third and final step performed in Section 3.4. The employed Leonov function candidate is presented in Section 3.2 and the derived conditions are discussed in Section 3.5.

\subsection{Local stability and error coordinates}

As existence of isolated equilibria is a natural constraint for a stability analysis, we make the following assumption.

Assumption 6 Condition (15) is satisfied with strict inequality.

Lemma 7 Consider the system (13) with Assumptions 1 and 6 . Then, the equilibrium $\eta_{0}^{s}$ in (16) is locally asymptotically stable. All other equilibria are unstable and the Jacobian of the dynamics (13) evaluated at any unstable equilibrium point has at least one eigenvalue with positive real part.
PROOF. Inspired by the proof of [31, Lemma 1], we observe that the Jacobian of the dynamics (13) can be written as the matrix product

$$
\mathcal{J}(\eta)=C \mathcal{H}(\eta) \in \mathbb{R}^{(2 N-1) \times(2 N-1)},
$$

where

$$
C=\left[\begin{array}{cc}
0 & \mathcal{B}^{\top} M^{-1} \\
-M^{-1} \mathcal{B} & -K^{-1} M^{-2}
\end{array}\right], \mathcal{H}(\eta)=\left[\begin{array}{cc}
\nabla^{2} U(\eta) & 0 \\
0 & M
\end{array}\right]
$$

and $\nabla^{2} U(\eta)$ is a diagonal matrix, see (12).

Recall that $K>0, M>0$ and that with Assumption 1 $\mathcal{B}$ has full column rank. Hence,

$$
\operatorname{det}(C)=\operatorname{det}\left(-K^{-1} M^{-2}\right) \operatorname{det}\left(-\mathcal{B}^{\top} K \mathcal{B}\right) \neq 0
$$

which shows that $C$ is invertible. Moreover, Assumption 6 implies that for any $\eta_{0}^{s, i}, i=1, \ldots, 2^{(N-1)}$, the matrix $\mathcal{H}\left(\eta_{0}^{s, i}\right)$ is invertible. Therefore, the matrix $\mathcal{J}\left(\eta_{0}^{s, i}\right)$ has no zero eigenvalue.

Since $\mathcal{J}(\eta)$ is asymmetric, we also need to show that $\mathcal{J}\left(\eta_{0}^{s, i}\right)$ has no other eigenvalues on the imaginary axis. This claim is established by contradiction. Let $v=\operatorname{col}\left(v_{1}, v_{2}\right), v_{1} \in \mathbb{C}^{(N-1)}, v_{2} \in \mathbb{C}^{N}$, be the eigenvector associated to a non-zero eigenvalue $\lambda \in \mathbb{R}$. Then,

$$
\begin{aligned}
\lambda v_{1} & =\mathcal{B}^{\top} v_{2} \\
\lambda v_{2} & =-M^{-1} \mathcal{B} \nabla_{\eta}^{2} U\left(\eta_{0}^{s, i}\right) v_{1}-M^{-1} K^{-1} v_{2}
\end{aligned}
$$

Since $\lambda \neq 0$, we obtain from the first equation $v_{1}=$ $\lambda^{-1} \mathcal{B}^{\top} v_{2}$. Inserting this in the second expression of (20), rearranging terms and multiplying with $v_{2}^{*} M \lambda$ from the left gives

$$
v_{2}^{*} M v_{2} \lambda^{2}+v_{2}^{*} K^{-1} v_{2} \lambda+v_{2}^{*} \mathcal{B} \nabla_{\eta}^{2} U\left(\eta_{0}^{s, i}\right) \mathcal{B}^{\top} v_{2}=0,
$$

which is a quadratic equation in $\lambda$. Since $K>0$, we have that $v_{2}^{*} K^{-1} v_{2} \neq 0$ for all $v_{2} \in \mathbb{C}^{N}$. Hence, it follows that the matrix $\mathcal{J}\left(\eta_{0}^{s, i}\right)$ has no purely imaginary eigenvalues.

Let $H\left(\eta_{0}^{s, i}\right)=-\mathcal{H}^{-1}\left(\eta_{0}^{s, i}\right)$ and note that

$$
\mathcal{J}\left(\eta_{0}^{s, i}\right) H\left(\eta_{0}^{s, i}\right)+H\left(\eta_{0}^{s, i}\right) \mathcal{J}^{\top}\left(\eta_{0}^{s, i}\right)=C+C^{\top} \geq 0
$$

Thus, by invoking [60, Lemma 2] we conclude that the number of eigenvalues with negative respectively positive real parts of the matrix $\mathcal{J}\left(\eta_{0}^{s, i}\right)$ is identical to the number of eigenvalues with negative respectively positive real parts of the matrix $-\mathcal{H}\left(\eta_{0}^{s, i}\right)$ (since the eigenvalues of $-\mathcal{H}^{-1}\left(\eta_{0}^{s, i}\right)$ have the same properties). 
For any $x \in \mathbb{R}^{(N-1)},\|x\|_{\infty}<1, \arcsin (x) \in$ $\left(-\frac{\pi}{2}, \frac{\pi}{2}\right)^{(N-1)}$, it follows immediately from (12) and (17) that all eigenvalues of $\nabla U\left(\eta_{0}^{s}\right)$ are positive real. This together with the fact that $M>0$ implies that all eigenvalues of $-\mathcal{H}\left(\eta_{0}^{s}\right)$ are negative real. Consequently, the equilibrium point $\operatorname{col}\left(\eta_{0}^{s}, \underline{0}_{N}\right)$ is locally asymptotically stable [47]. From (16) we see that any other $\eta_{0}^{s, i} \neq \eta_{0}^{s}$ has at least one entry, say the $k$-th, satisfying

$$
\hat{\eta}_{0 k}^{s}=\left(\pi-\eta_{0 k}^{s}\right) \in\left(\frac{\pi}{2}, \frac{3 \pi}{2}\right) .
$$

Consequently, $-\mathcal{H}\left(\eta_{0}^{s, i}\right)$ has at least one positive real eigenvalue. Therefore, all equilibria with $\eta_{0}^{s, i} \neq \eta_{0}^{s}$ are unstable [47], completing the proof.

With Assumption 6, we denote the asymptotically stable equilibrium point of the system (13) by $\operatorname{col}\left(\eta^{*}, \mathbb{1}_{N} \omega^{*}\right)$, i.e., $\eta^{*}=\eta_{0}^{s}$, and introduce the error states

$\tilde{\eta}(t)=\eta(t)-\eta^{*} \in \mathbb{R}^{(N-1)}, \quad \tilde{\omega}(t)=\omega(t)-\mathbb{1}_{N} \omega^{*} \in \mathbb{R}^{N}$, $\tilde{x}(t)=\operatorname{col}(\tilde{\eta}(t), \tilde{\omega}(t))$

as well as the short-hand

$$
\zeta(\tilde{\eta})=\nabla U\left(\tilde{\eta}+\eta^{*}\right)-\nabla U\left(\eta^{*}\right) .
$$

Then, in error coordinates, the system (13) becomes

$$
\begin{aligned}
\dot{\tilde{\eta}} & =\mathcal{B}^{\top} \tilde{\omega}, \\
M \dot{\tilde{\omega}} & =-K^{-1} \tilde{\omega}-\mathcal{B} \zeta(\tilde{\eta}),
\end{aligned}
$$

the equilibrium of which is now shifted to the origin and, because of Assumption 6 and Lemma 7, the origin is isolated and asymptotically stable. The remainder of this section is devoted to the analysis of this system.

\subsection{Leonov function candidate}

The notion of a Leonov function is introduced following $[46,1]$. For its presentation, we define three auxiliary sets:

$$
\begin{aligned}
\mathcal{W}:= & \left\{\operatorname{col}(\tilde{\eta}, \tilde{\omega}) \in \mathbb{R}^{(2 N-1)}:|\tilde{\eta}|_{\infty}=c\right\}, \pi \leq c<2 \pi, \\
\mathcal{U}:= & \cup_{r \in \mathbb{Z}_{\geq 0}} \mathcal{U}_{r}, \\
\mathcal{U}_{r}:= & \left\{\operatorname{col}(\tilde{\eta}, \tilde{\omega}) \in \mathbb{R}^{(2 N-1)}: \tilde{\omega}=\underline{0}_{N},|\tilde{\eta}|_{\infty}=2 r \pi,\right. \\
& \left.\mathcal{B} \zeta(\tilde{\eta})=\underline{0}_{N}\right\} .
\end{aligned}
$$

Since the microgrid dynamics $(22)$ are $2 \pi$-periodic in $\tilde{\eta}$, the definitions of the variable $r$ and of the set $\mathcal{U}_{r}$, ensure that the set $\mathcal{U}$ includes all equilibria of the system obtained by shifting the one at the origin. This is essential for the concept of a Leonov function for the system (22), which is defined as follows $[46,1]$.
Definition 8 ([1]) $A \mathcal{C}^{1}$ function $V: \mathbb{R}^{(2 N-1)} \rightarrow \mathbb{R}$ is a Leonov function for the system (22) if there exist a constant $g \geq 0$, functions $\alpha \in \mathcal{K}_{\infty}, \psi \in \mathcal{K}$ and a continuous function $\lambda: \mathbb{R} \rightarrow \mathbb{R}$, satisfying $\lambda(0)=0$ and $\lambda(s) s>0$ for all $s \neq 0$, such that

$$
\begin{aligned}
& \alpha(|\tilde{\omega}|)-\psi(|\tilde{\eta}|)-g \leq V(\tilde{\eta}, \tilde{\omega}) \forall \operatorname{col}(\tilde{\eta}, \tilde{\omega}) \in \mathbb{R}^{(2 N-1)}, \\
& \inf _{\operatorname{col}(\tilde{\eta}, \tilde{\omega}) \in \mathcal{W}} V(\tilde{\eta}, \tilde{\omega})>0, \sup _{\operatorname{col}(\tilde{\eta}, \tilde{\omega}) \in \mathcal{U}} V(\tilde{\eta}, \tilde{\omega}) \leq 0
\end{aligned}
$$

and the following dissipation inequality holds:

$$
\dot{V}+\lambda(V) \leq 0 \quad \forall \operatorname{col}(\tilde{\eta}, \tilde{\omega}) \in \mathbb{R}^{(2 N-1)} .
$$

From (24) and (25), we see that the sign definiteness requirements of a Leonov function are relaxed compared to a standard Lyapunov function [48], because the function $V$ in Definition 8 does not have to be positive definite with respect to the variable $\tilde{\eta}$, i.e., the variable with respect to which the dynamics (22) are periodic. Furthermore, the time-derivative of $V$ only needs to be negative definite for positive values of $V$. See [46,1] for further details.

The requirements on (25) have been further relaxed in [1, Corollary 3]. This result is used to establish the main result of the present paper and hence recalled here. For this purpose, we introduce the following sets:

$$
\begin{aligned}
\Omega & =\left\{\operatorname{col}(\tilde{\eta}, \tilde{\omega}) \in \mathbb{R}^{(2 N-1)}: V \leq 0\right\}, \\
\Omega_{\varepsilon, c}^{\prime} & =\left\{\operatorname{col}(\tilde{\eta}, \tilde{\omega}) \in \mathbb{R}^{(2 N-1)}: V \leq \varepsilon,|\tilde{\eta}|_{\infty}<c\right\}, \\
\mathcal{Z} & =\left\{\operatorname{col}(\tilde{\eta}, \tilde{\omega}) \in \mathbb{R}^{(2 N-1)}:|\tilde{\omega}|>\xi\right\},
\end{aligned}
$$

with $c$ defined in (23) and for some $\varepsilon \in \mathbb{R}_{>0}$ and $\xi \in \mathbb{R}_{>0}$.

Corollary 9 ([1]) Suppose that there exists a Leonov function $V: \mathbb{R}^{(2 N-1)} \rightarrow \mathbb{R}$ for the system (22), such that $\sup _{\tilde{\eta} \in \mathbb{R}^{(N-1)}} \psi(|\tilde{\eta}|)<+\infty$ and the inequality $(25)$ is verified only for $\operatorname{col}(\tilde{\eta}, \tilde{\omega}) \in\left(\mathbb{R}^{(2 N-1)} \backslash \Omega\right) \cap\left(\mathcal{Z} \cup \Omega_{\varepsilon, c}^{\prime}\right)$. Then for all initial conditions $\operatorname{col}(\tilde{\eta}(0), \tilde{\omega}(0)) \in \mathbb{R}^{(2 N-1)}$ the corresponding trajectories $\operatorname{col}(\tilde{\eta}, \tilde{\omega})$ are bounded $\forall t \geq 0 . \square$

Let

$$
h(\tilde{\eta}, \tilde{\omega})=\alpha K^{-1} \tilde{\omega}+\mathcal{B} \zeta(\tilde{\eta}),
$$

with $\alpha \in[0,1]$ and $\zeta(\tilde{\eta})$ defined in (21). By direct calculations we obtain

$\frac{d}{d t} h=-\alpha K^{-1} M^{-1} h+\left(\alpha(\alpha-1) K^{-2} M^{-1}+\mathcal{B} S\left(\tilde{\eta}+\eta^{*}\right)\right) \tilde{\omega}$,

where

$$
S\left(\tilde{\eta}+\eta^{*}\right)=\nabla^{2} U\left(\tilde{\eta}+\eta^{*}\right) \mathcal{B}^{\top}=A \cos \left(\tilde{\eta}+\eta^{*}\right) \mathcal{B}^{\top} .
$$




$$
Q(\tilde{\eta})=\left[\begin{array}{cc}
\alpha\left(\Phi K^{-1} M^{-1}+K^{-1} M^{-1} \Phi\right) & -\Phi\left(\alpha(\alpha-1) K^{-2} M^{-1}+\mathcal{B} S\left(\tilde{\eta}+\eta^{*}\right)\right) \\
\left(-\Phi\left(\alpha(\alpha-1) K^{-2} M^{-1}+\mathcal{B} S\left(\tilde{\eta}+\eta^{*}\right)\right)\right)^{\top} & 2 K^{-1}-(\nu+\mu) I_{N}
\end{array}\right]-\beta\left[\begin{array}{cc}
I_{N} & -\alpha K^{-1} \\
-\alpha K^{-1} & \alpha^{2} K^{-2}
\end{array}\right]
$$

Let $\kappa \in \mathbb{R}_{\geq 0}$ be a parameter and $\Phi \in \mathbb{R}^{N \times N}$, $\Phi=\Phi^{\top}>0$, be a design matrix. Then our proposed Leonov function candidate for the system (22) is

$$
\begin{aligned}
V(\tilde{\eta}, \tilde{\omega})= & \tilde{\omega}^{\top} M \tilde{\omega}+h^{\top}(\tilde{\eta}, \tilde{\omega}) \Phi h(\tilde{\eta}, \tilde{\omega})-\kappa \\
& +2\left[U\left(\tilde{\eta}+\eta^{*}\right)-U\left(\eta^{*}\right)-\nabla U^{\top}\left(\eta^{*}\right) \tanh (\tilde{\eta})\right] \\
= & {\left[\begin{array}{c}
\mathcal{B} \zeta(\tilde{\eta}) \\
\tilde{\omega}
\end{array}\right]^{\top} \underbrace{\left[\begin{array}{cc}
\Phi & \alpha \Phi K^{-1} \\
\alpha K^{-1} \Phi & M+\alpha^{2} K^{-1} \Phi K^{-1}
\end{array}\right]}_{:=\Psi}\left[\begin{array}{c}
\mathcal{B} \zeta(\tilde{\eta}) \\
\tilde{\omega}
\end{array}\right] } \\
& +2\left[U\left(\tilde{\eta}+\eta^{*}\right)-U\left(\eta^{*}\right)-\nabla U^{\top}\left(\eta^{*}\right) \tanh (\tilde{\eta})\right] \\
& -\kappa .
\end{aligned}
$$

We also make use of this lower bound for $V$ in the sequel:

$$
\begin{aligned}
\underline{V}(\tilde{\eta})= & 2\left[U\left(\tilde{\eta}+\eta^{*}\right)-U\left(\eta^{*}\right)-\nabla U^{\top}\left(\eta^{*}\right) \tanh (\tilde{\eta})\right] \\
& +\lambda_{\min }(\Psi)|\mathcal{B} \zeta(\tilde{\eta})|^{2}-\kappa \leq V \quad \forall(\tilde{\eta}, \tilde{\omega}) \in \mathbb{R}^{(2 N-1)}
\end{aligned}
$$

and, in particular, of its behavior in the set

$$
\underline{\Omega}=\left\{\operatorname{col}(\tilde{\eta}, \tilde{\omega}) \in \mathbb{R}^{(2 N-1)}: \underline{V} \leq 0,0<|\tilde{\eta}|_{\infty} \leq c\right\} .
$$

\subsection{A condition for global boundedness of solutions}

A sufficient condition for global boundedness of trajectories is presented by deriving conditions under which $V$ in (31) is a Leonov function for the dynamics (22). To streamline the presentation of our result the following assumption is needed.

Assumption 10 Recall the sets $\mathcal{W}$ and $\underline{\Omega}$ and the function $\underline{V}$ defined in (23), (33) and (32), respectively. Consider the matrix $Q(\tilde{\eta})$ given in $(27)$. There exist parameters $K>0, c \in[\pi, 2 \pi), \Phi>0, \alpha \in[0,1], \nu>0, \beta>0$ and $\mu>0$, such that

$$
\begin{aligned}
& \inf _{\operatorname{col}(\tilde{\eta}, \tilde{\omega}) \in \mathcal{W}} \underline{V}(\tilde{\eta})>0 \\
& \\
& \quad Q(\tilde{\eta}) \geq 0 \\
& \sup _{\operatorname{col}\left(\tilde{\eta}, \underline{\underline{n}}_{N}\right) \in \underline{\beta}}|\mathcal{B} \zeta(\tilde{\eta})|^{2}+\frac{1}{\nu}\left|\mathcal{B} \operatorname{diag}\left(\tanh ^{2}(\tilde{\eta})\right) \nabla U\left(\eta^{*}\right)\right|^{2}<0 .
\end{aligned}
$$

Proposition 11 Consider the system (22) with Assumptions 1, 6 and 10. Select

$$
\kappa=2 \sum_{i=1}^{N-1}\left|a_{i} \sin \left(\eta_{i}^{*}\right)\right|
$$

Then, the function $V$ in (31) is a Leonov function for the system (22). Furthermore, all solutions of the system (22) are bounded.

PROOF. The claim is established by invoking Corollary 9. Recall the sets $\mathcal{W}$ and $\mathcal{U}$ defined in (23) as well as $\Omega_{\varepsilon, c}^{\prime}$ in $(26)$. Since $\tanh (\tilde{\eta}) \in[-1,1]^{(N-1)}$, by choosing $\kappa$ as specified in (35), we ensure that $\sup _{\operatorname{col}(\tilde{\eta}, \tilde{\omega}) \in \mathcal{U}} V \leq$

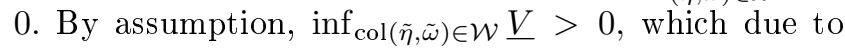
(32) implies that $\inf _{\operatorname{col}(\tilde{\eta}, \tilde{\omega}) \in \mathcal{W}} V>0$. Furthermore, it can be seen from (31) in a straightforward manner that the required functions $\alpha \in \mathcal{K}_{\infty}$ and $\psi \in \mathcal{K}$ as well as the constant $g \geq 0$ exist for the proposed function $V$. Hence, the conditions in (24) are satisfied. Moreover, since $U\left(\tilde{\eta}+\eta^{*}\right), \tanh (\tilde{\eta})$ and $\zeta(\tilde{\eta})$ are bounded functions, the additional requirement $\sup _{\tilde{\eta} \in \mathbb{R}^{(N-1)}} \psi(|\tilde{\eta}|)<+\infty$ of Corollary 9 is satisfied.

Next, with (29), (30) and

$$
\frac{d}{d t} \tanh (\tilde{\eta})=\operatorname{diag}\left(\mathbb{1}_{(N-1)}-\tanh ^{2}(\tilde{\eta})\right) \dot{\tilde{\eta}}
$$

we have that

$$
\begin{aligned}
\dot{V}= & -2 \tilde{\omega}^{\top} K^{-1} \tilde{\omega}-2 \tilde{\omega}^{\top} \mathcal{B} \zeta(\tilde{\eta})+2 \nabla U^{\top}\left(\tilde{\eta}+\eta^{*}\right) \mathcal{B}^{\top} \tilde{\omega} \\
& -2 \nabla U^{\top}\left(\eta^{*}\right) \operatorname{diag}\left(\mathbb{1}_{N-1}-\tanh ^{2}(\tilde{\eta})\right) \mathcal{B}^{\top} \tilde{\omega} \\
& -2 \alpha h^{\top} \Phi K^{-1} M^{-1} h \\
& +2 h^{\top} \Phi\left(\alpha(\alpha-1) K^{-2} M^{-1}+\mathcal{B} S\left(\tilde{\eta}+\eta^{*}\right)\right) \tilde{\omega} \\
\leq & -\tilde{\omega}^{\top}\left(2 K^{-1}-\nu I_{N}\right) \tilde{\omega}+\frac{1}{\nu}\left|\mathcal{B} \operatorname{diag}\left(\tanh ^{2}(\tilde{\eta})\right) \nabla U\left(\eta^{*}\right)\right|^{2} \\
& -2 \alpha h^{\top} \Phi K^{-1} M^{-1} h \\
& +2 h^{\top} \Phi\left(\alpha(\alpha-1) K^{-2} M^{-1}+\mathcal{B} S\left(\tilde{\eta}+\eta^{*}\right)\right) \tilde{\omega} \\
= & -\left[\begin{array}{l}
h \\
\tilde{\omega}
\end{array}\right]^{\top} Q(\tilde{\eta})\left[\begin{array}{l}
h \\
\tilde{\omega}
\end{array}\right]-\mu\left|\tilde{\omega}^{2}\right|-\beta|\mathcal{B} \zeta(\tilde{\eta})|^{2} \\
& +\frac{1}{\nu}\left|\mathcal{B} \operatorname{diag}\left(\tanh ^{2}(\tilde{\eta})\right) \nabla U\left(\eta^{*}\right)\right|^{2} \\
\leq & -\mu\left|\tilde{\omega}^{2}\right|-\beta|\mathcal{B} \zeta(\tilde{\eta})|^{2}+\frac{1}{\nu}\left|\mathcal{B} \operatorname{diag}\left(\tanh ^{2}(\tilde{\eta})\right) \nabla U\left(\eta^{*}\right)\right|^{2},
\end{aligned}
$$


where $Q(\tilde{\eta})$ is given in (27), $\nu, \mu$ as well as $\beta$ are positive parameters and the last inequality follows since $Q(\tilde{\eta}) \geq 0$ by assumption.

With the above relations, it is difficult to ensure that condition $(25)$ is satisfied for all $\operatorname{col}(\tilde{\eta}, \tilde{\omega}) \in \mathbb{R}^{(2 N-1)}$. Therefore, we employ the relaxed requirement of Corollary 9 instead and, thus, investigate the behavior of $\dot{V}$ restricted to arguments $\operatorname{col}(\tilde{\eta}, \tilde{\omega}) \in\left(\mathbb{R}^{(2 N-1)} \backslash \Omega\right) \cap\left(\mathcal{Z} \cup \Omega_{\varepsilon}\right)$. Recall that $Q(\tilde{\eta}) \geq 0$ by assumption, both $\zeta$ and tanh are bounded functions, $\mu>0$ and $\nabla U\left(\eta^{*}\right)$ is a constant. Hence, it is evident that there exist $\xi>0$ and $\chi>0$, such that $\dot{V}+\chi V \leq 0$ for all $\operatorname{col}(\tilde{\eta}, \tilde{\omega}) \in \mathcal{Z}$ defined in (26).

Determining both the set $\Omega_{\varepsilon, c}^{\prime}$ defined in (26) and the behavior of $\dot{V}$ on this set explicitly may be difficult. Yet, it follows from (36) that the last inequality in (34) and the fact that $\dot{V} \leq 0$ for all $\operatorname{col}\left(\underline{0}_{N-1}, \tilde{\omega}\right) \in \mathbb{R}^{(2 N-1)}$ imply that $\dot{V} \leq 0$ for all $(\tilde{\eta}, \tilde{\omega}) \in \underline{\Omega}$ with $\underline{\Omega}$ defined in (33). Since the last inequality in (34) is strict, then for some $\varepsilon>0$ the same inequality is satisfied for $\frac{V}{1)}=\varepsilon$. Furthermore, since $\underline{V} \leq V$ for all $(\tilde{\eta}, \tilde{\omega}) \in \mathbb{R}^{(2 N-\overline{1)})}$, with $\underline{V}$ defined in (32), we have that

$$
\left(\Omega_{0, c}^{\prime} \backslash\left\{\underline{0}_{(2 N-1)}\right\}\right) \subseteq \underline{\Omega} .
$$

Consequently, by continuity of $V$ and $\underline{V}$ there exist (sufficiently small) parameters $\varepsilon>0$ and $\chi>0$, such that $\dot{V} \leq-\chi V$ for all $\Omega_{\varepsilon, c}^{\prime}$.

Thus, all conditions of Corollary 9 are satisfied. Hence, $V$ is a Leonov function for the system (22) and all solutions $\operatorname{col}(\tilde{\eta}, \tilde{\omega}) \in \mathbb{R}^{(2 N-1)}$ are bounded for all $t \geq 0$.

\subsection{Main synchronization result}

Recall from Section 3.1 that under Assumption 6, Lemma 7 implies that the dynamics (22) have one asymptotically stable equilibrium point and $2^{(N-1)}-1$ unstable equilibria (modulo $2 \pi$ ). Denote the set of asymptotically stable equilibria of the system (22) by $\mathcal{X}$.

We now show that the conditions of Proposition 11 also imply almost global asymptotic stability of the set $\mathcal{X}$.

Theorem 12 Consider the system (22) with Assumptions 1, 6 and 10. Then, the set $\mathcal{X}$ is almost globally asymptotically stable, i.e., for all initial conditions, except a set of measure zero, the solutions of the system (22) asymptotically converge to a point in $\mathcal{X}$.

PROOF. Recall that with Assumptions 1, 6 and 10, Proposition 11 implies that all solutions of the system
(22) are bounded. Thus, it only remains to show that almost all bounded solutions converge to a stable equilibrium point. To this end, consider the function ${ }^{4} W$ : $\mathbb{R}^{(2 N-1)} \rightarrow \mathbb{R}$

$$
W(\tilde{\eta}, \tilde{\omega})=\frac{1}{2} \tilde{\omega}^{\top} M \tilde{\omega}+U\left(\tilde{\eta}+\eta^{*}\right)-\nabla U^{\top}\left(\eta^{*}\right) \tilde{\eta} .
$$

With (22), a straightforward calculation yields

$$
\dot{W}=-\tilde{\omega}^{\top} K^{-1} \tilde{\omega} \leq 0 .
$$

Consequently, by invoking LaSalle's invariance principle [48] we conclude that all bounded solutions of the system (22) converge to the set where

$$
\tilde{\omega}(t)=\underline{0}_{N}, \quad \forall t \in \mathbb{R}_{\geq 0} .
$$

By inspection of (22), we see that this implies that $\tilde{\eta}$ is constant. Consequently, the invariant set where $\dot{W} \equiv 0$ contains all equilibria of the system (22). This shows that for all initial conditions, the solutions of the system (22) asymptotically converge to an equilibrium.

To show that the set $\mathcal{X}$ is almost globally asymptotically stable, we note that Lemma 7 implies that the Jacobian of the dynamics (22) evaluated at any unstable equilibrium point has at least one eigenvalue with positive real part. Thus, following the analyses in $[44,45,52]$, we invoke [61, Proposition 11] to conclude that the region of attraction of any unstable equilibrium point has zero Lebesgue measure. Hence, for all initial conditions, except a set of measure zero, the solutions of the system (22) asymptotically converge to an isolated point in the set $\mathcal{X}$, completing the proof.

As discussed at the end of Section 2.2, almost global asymptotic stability of the set $\mathcal{X}$ is equivalent to global asymptotic convergence of almost all solutions $\operatorname{col}(\theta, \omega)$ to $\operatorname{col}\left(\theta^{s}, \omega^{s}\right)$, modulo $2 \pi$ and up to a constant uniform shift $\theta_{\mathrm{av}} \mathbb{1}_{N}, \theta_{\mathrm{av}} \in \mathbb{R}$, in all angles.

\subsection{Discussion on the synchronization conditions}

In order to illustrate the implications of our main convergence condition, i.e., Assumption 10, we simplify the matrix $Q(\tilde{\eta})$ given in (27) - at the cost of potentially more conservative conditions. To this end, we need the following result.

Define the constant

$$
\rho:=\sup _{\tilde{\eta} \in \mathbb{R}^{(N-1)}}\left\|\mathcal{B} S\left(\tilde{\eta}+\eta^{*}\right)\right\|_{2}
$$

\footnotetext{
${ }^{4}$ Note that $W$ is continuously differentiable as a function $\mathbb{R}^{(2 N-1)} \rightarrow \mathbb{R}$, but not as a function $\mathbb{S}^{(N-1)} \times \mathbb{R}^{N} \rightarrow \mathbb{R}$
} 
where $\|\cdot\|_{2}$ is the induced 2-matrix-norm. The proof of the lemma below is given in Appendix A.

Lemma 13 The constant $\rho$ is given by

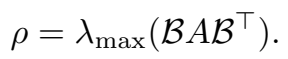

Now we can establish the following claim, which-for clarity of exposition - is derived for the case of uniform (virtual) inertia constants and droop gains. The corresponding proof is presented in Appendix B.

Lemma 14 Suppose that $K=k I_{N}, M=m I_{N}, k>0$, $m>0$ and $m$ is fixed. Set $\beta=k$ and $\alpha=1$ and select $k$ and $\nu$, such that

$$
1-\nu k-m^{2} \rho^{2} k^{4}-2 m \rho k^{2}>0,
$$

where the constant $\rho$ is given in (40). Then $Q(\tilde{\eta})>0$ for all $\eta^{*} \in \mathbb{R}^{(N-1)}$ and all $\tilde{\eta} \in \mathbb{R}^{(N-1)}$ as well as some $\mu>0$.

Based on Lemma 14 we can draw the following physical implications of our main result, i.e., Theorem 12:

- The constant $\rho$ defined in (39) can be interpreted as an upper bound on the network interconnection strength represented by the coefficients $a_{i}>0$ in $\mathcal{B} \nabla U\left(\tilde{\eta}+\eta^{*}\right) \mathcal{B}^{\top}$, see (5) and Lemma 13. For the particular case of uniform coupling coefficients, i.e., $a_{i}=\bar{a}$, $\bar{a}>0, i \sim \mathcal{N}$, we have that $\rho=\bar{a} \lambda_{\max }\left(\mathcal{B B}^{\top}\right)$, where $\lambda_{\max }\left(\mathcal{B B}^{\top}\right)$ denotes the largest eigenvalue of the unweighted Laplacian matrix of the network graph.

- Condition (41) reveals that the requirement $Q(\tilde{\eta}) \geq$ 0 can always be satisfied for any equilibrium $\eta^{*} \in$ $\mathbb{R}^{(N-1)}$ by choosing a sufficiently small droop coefficient $k$.

- Condition (41) also shows that the larger the inertia constants $m$ and the parameter $\rho$ are, the smaller the droop coefficients $k$ have to be for Assumption 10 to be satisfied. In particular with decreasing inertia coefficients, i.e., $m \rightarrow 0$, condition (41) reduces to $1-\nu k>0$. In other words, the conditions (27) and (41) are more likely to be satisfied for loosely interconnected MGs in which the units have low (virtual) inertia constants and are strongly damped.

- The following "Lyapunov-like" interpretation of the role of the interconnection strength is in order. If the angle differences are less than $\frac{\pi}{2}$, then the Hessian $\nabla^{2} U$ is positive definite. Hence, the solutions are already in a good "valley" of the state space and therefore strong network links (i.e., large $\rho$ ) help to stay in that region and eventually converge. However, if some angle differences are larger than $\frac{\pi}{2}$ and the corresponding $a_{i}$ are strong it might be difficult for the

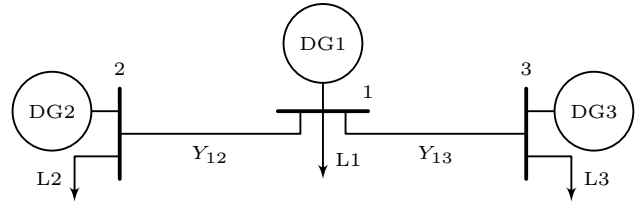

Fig. 1. Schematic representation of the considered MG test system based on data in [29].

system to "climb" over the saddle and make it over to the "good" region. Therefore, in any local setting strong links are good, whereas in a global setting they are not.

- The requirement $Q(\tilde{\eta})>0$ is necessary for Assumption 10 to hold, but not sufficient. The other two conditions in Assumption 10 are equilibrium-dependent. Note in particular that by construction the set $\mathcal{W}$ cannot contain any equilibrium point of (22) since in such a case the derivative of $V$ would be strictly negative at an equilibrium. Hence, the set $\mathcal{W}$ separates the equilibria of the system and the condition

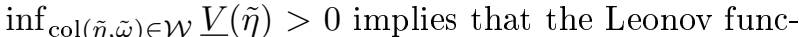
tion is negative definite with respect to the distance to the set $\mathcal{W}$. This property is essential for the derivation of the multivariable cell structure approach in [1].

- With regard to the third and last condition in Assumption 6 , physically the term $\mathcal{B} \nabla U\left(\eta^{*}\right)$ corresponds to the stationary network power flows. Thus, we see from the property of the tanh-function together with the fact that $1-\beta \nu>0$ (see (41)) that the conditions for global boundedness of trajectories in Proposition 11 are more likely to be satisfied in lightly loaded operating conditions. This seems reasonable from a practical point of view.

\section{Numerical example}

The analysis is illustrated on a MG based on the Subnetwork 1 of the CIGRE MV benchmark model [29]. For this purpose, we assume all breakers in the system are open and only consider three nodes to have DG units (namely, the nodes nodes 5, 9 and 10 in [29]). The system topology is shown in Fig. 1 and the corresponding data is given in Table 1. In Section 4.1 we show how the derived almost global synchronization conditions in Assumption 10 can be evaluated graphically, while in Section 4.2 we propose a numerical evaluation procedure.

\subsection{Graphical evaluation procedure for the synchroniza- tion conditions}

The conditions in Assumption 10 are evaluated graphically for a range of operating points. At first we determine the parameters $\alpha, \beta, \nu$ and $\Phi$, such that $Q(\tilde{\eta}) \leq 0$. 
Table 1

Main test system parameters in per unit (pu)

\begin{tabular}{ll}
\hline Base values & $S_{\text {base }}=1[\mathrm{MW}], V_{\text {base }}=20[\mathrm{kV}]$ \\
DG power ratings & $S_{N}=[2.53,2.09,0.86][\mathrm{pu}]$ \\
Admittances & $Y_{12}=22.31[\mathrm{pu}], Y_{13}=12.74[\mathrm{pu}]$ \\
$\begin{array}{l}\text { Droop gains } \\
\begin{array}{l}\text { Virtual inertia } \\
\text { constants }\end{array}\end{array}$ & $K=0.05 \operatorname{diag}\left(S_{N}\right)^{-1}\left[\frac{\mathrm{Hz}}{\mathrm{pu}}\right]$ \\
\hline
\end{tabular}

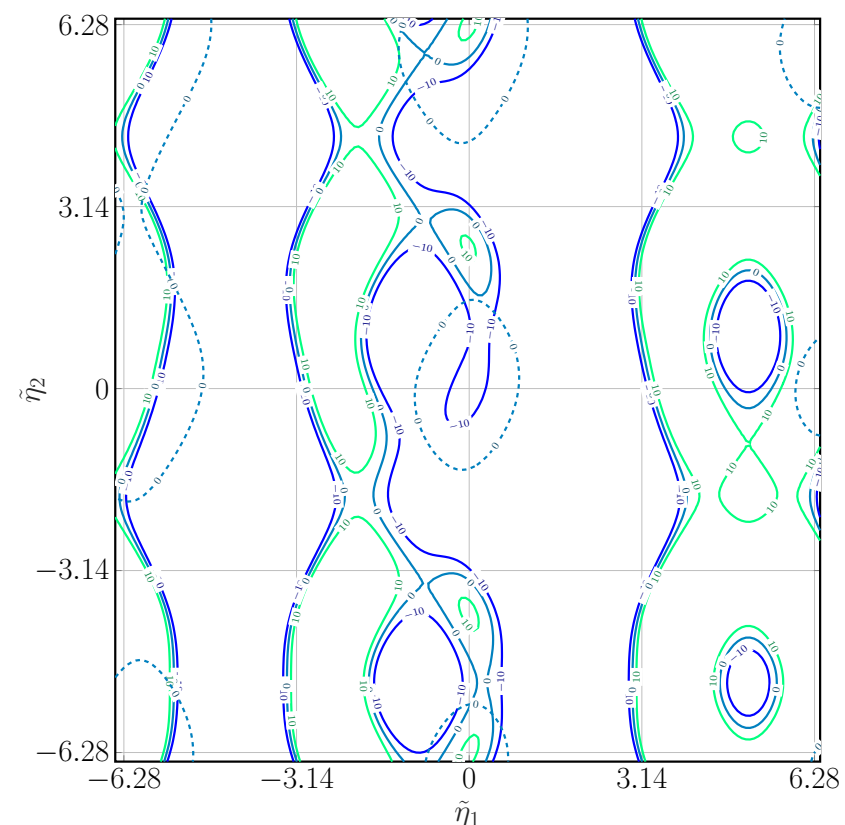

Fig. 2. Contour plot of $-\beta\left|\mathcal{B} \zeta\left(\tilde{\eta}+\eta^{*}\right)\right|^{2}+\frac{1}{\nu}\left|\mathcal{B} \operatorname{diag}\left(\tanh ^{2}(\tilde{\eta})\right) \nabla U\left(\eta^{*}\right)\right|^{2}$ for $|\tilde{\eta}|_{\infty} \leq 2 \pi$ and $\eta^{*}=\operatorname{col}\left(20^{\circ},-21^{\circ}\right)$. The dashed curves represent the level set $V=0$. The plots show that with $c=\pi$ the conditions on $\underline{\Omega}$ and $\mathcal{W}$ in Assumption 10 are satisfied.

From Assumption 10 it is evident that we seek to maximize $\beta$ and $\nu$. This is done via a polytopic approach and by implementing the corresponding matrix inequalities in Yalmip [62] with $\mu=0$ and $\alpha=1$. With the given system data, we obtain $\beta=0.12$ and $\nu=3.49$

Next we evaluate the feasibility of the remaining two conditions in Assumption 10 for a wide range of different operating points. We find that the conditions are feasible for values up to $\left|\eta^{*}\right|_{\infty}=21^{\circ}$. This shows that a reasonable range of operating points can be guaranteed to be almost globally asymptotically stable with the conditions of Proposition 11. In Fig. 2 the contour plots corresponding to the functions appearing in the second and third conditions of Assumption 10 are shown for an exemplary operating point with $\eta^{*}=\operatorname{col}\left(20^{\circ},-21^{\circ}\right)$. It can be seen that for $\mathcal{W}$ in (23) with $c=\pi$, Assumption 10 is satisfied.

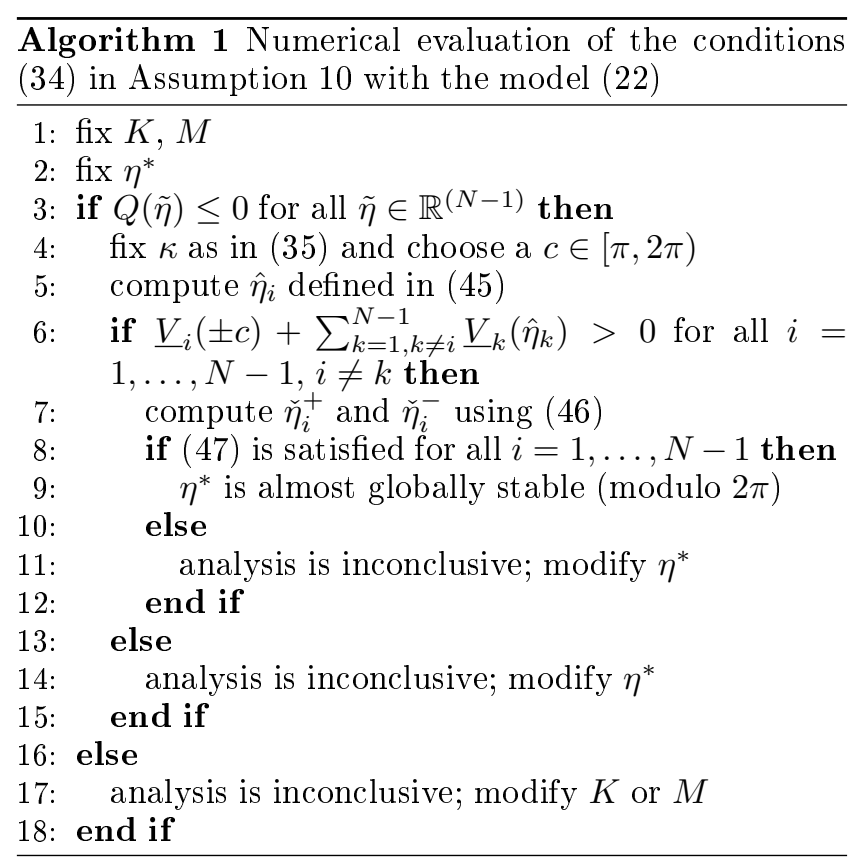

4.2 Numerical evaluation procedure for the synchronization conditions

For $N>3$, a graphical evaluation of the synchronization conditions as conducted in Section 4.1 is difficult. Therefore, we propose Algorithm 1 as an alternative numerical evaluation procedure. The algorithm is derived as follows. Recall (34) in Assumption 10 and note that $\underline{V}$ in $(32)$ can be written element-wise in terms of $\tilde{\eta}_{i}$, i.e.,

$$
\underline{V}(\tilde{\eta})=\sum_{i=1}^{N-1} \underline{V}_{i}\left(\tilde{\eta}_{i}\right),
$$

with

$$
\begin{aligned}
\underline{V}_{i}\left(\tilde{\eta}_{i}\right)= & 2 a_{i}\left(-\cos \left(\tilde{\eta}_{i}+\eta_{i}^{*}\right)+\cos \left(\eta_{i}^{*}\right)-\sin \left(\eta_{i}^{*}\right) \tanh \left(\tilde{\eta}_{i}\right)\right) \\
& -\kappa_{i}+\lambda_{\min }(\Psi) a_{i}^{2}\left(\sin \left(\tilde{\eta}_{i}+\eta_{i}^{*}\right)-\sin \left(\eta_{i}^{*}\right)\right)^{2}
\end{aligned}
$$

Furthermore, we have that

$$
\begin{aligned}
& -\beta|\mathcal{B} \zeta(\tilde{\eta})|^{2}+\frac{1}{\nu}\left|\mathcal{B} \operatorname{diag}\left(\tanh ^{2}(\tilde{\eta})\right) \nabla U\left(\eta^{*}\right)\right|^{2} \\
\leq & -\beta \lambda_{\min }\left(\mathcal{B}^{\top} \mathcal{B}\right)|\zeta(\tilde{\eta})|^{2} \\
& +\frac{1}{\nu} \lambda_{\max }\left(\mathcal{B}^{\top} \mathcal{B}\right)\left|\operatorname{diag}\left(\tanh ^{2}(\tilde{\eta})\right) \nabla U\left(\eta^{*}\right)\right|^{2}
\end{aligned}
$$

The right hand-side of (43) is a system of $N-1$ decoupled equations in the variables $\tilde{\eta}_{i}$. Hence, a sufficient 
condition for the last inequality in (34) to be satisfied is

$$
\begin{aligned}
\sup _{\left(\tilde{\eta}, \underline{\theta}_{N}\right) \in \underline{\Omega}} & -\beta \lambda_{\min }\left(\mathcal{B}^{\top} \mathcal{B}\right)|\zeta(\tilde{\eta})|^{2} \\
& +\frac{1}{\nu} \lambda_{\max }\left(\mathcal{B}^{\top} \mathcal{B}\right)\left|\operatorname{diag}\left(\tanh ^{2}(\tilde{\eta})\right) \nabla U\left(\eta^{*}\right)\right|^{2}<0 .
\end{aligned}
$$

With these derivations, let

$$
\hat{\eta}_{i}:=\operatorname{argmin}_{\tilde{\eta}_{i},\left|\tilde{\eta}_{i}\right| \leq c} \underline{V}_{i}\left(\tilde{\eta}_{i}\right),
$$

which can be computed (numerically) explicitly for any given $\eta_{i}^{*}$ from (42) and corresponds to line 5 in Algorithm 1 . Then, for the first inequality in (34) to be satisfied we need that for each $i=1, \ldots, N-1$,

$$
\underline{V}_{i}( \pm c)+\sum_{k=1, k \neq i}^{N-1} \underline{V}_{k}\left(\hat{\eta}_{k}\right)>0
$$

which is included in line 6 in Algorithm 1.

To verify the last inequality in (34) numerically, we see

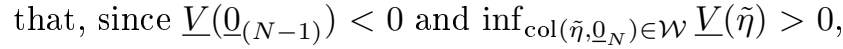
there has to exist at least one $\tilde{\eta}_{i} \in(0, c]$ and one in $[-c, 0)$, such that

$$
\underline{V}_{i}\left(\tilde{\eta}_{i}\right)+\sum_{k=1, k \neq i}^{N-1} \underline{V}_{k}\left(\hat{\eta}_{k}\right)=0,
$$

where $\hat{\eta}_{k}$ is defined in (45). Denote the solutions to this equality by $\check{\eta}_{i}^{-} \in[-c, 0)$ and $\check{\eta}_{i}^{+} \in(0, c]$ (if there is more than one solution on one of the intervals, choose the one closest to the origin). Then by using (43) a sufficient condition for the last inequality in (34) to be satisfied is

$$
\begin{aligned}
& -\beta \lambda_{\min }\left(\mathcal{B}^{\top} \mathcal{B}\right) a_{i}^{2}\left(\sin \left(\tilde{\eta}_{i}+\eta_{i}^{*}\right)-\sin \left(\eta_{i}^{*}\right)\right)^{2} \\
& +\frac{1}{\nu} \lambda_{\max }\left(\mathcal{B}^{\top} \mathcal{B}\right) \tanh ^{4}\left(\tilde{\eta}_{i}\right) a_{i}^{2} \sin \left(\eta_{i}^{*}\right)^{2}<0
\end{aligned}
$$

for all $\tilde{\eta}_{i} \in\left[\check{\eta}_{i}^{-}, \check{\eta}_{i}^{+}\right] \backslash\{0\}$. This corresponds to lines $7-8$ of Algorithm 1. By letting

$$
\underline{\Omega}^{\prime}=\left[\check{\eta}_{1}^{-}, \check{\eta}_{1}^{+}\right] \times \cdots \times\left[\check{\eta}_{N-1}^{-}, \check{\eta}_{N-1}^{+}\right] \backslash\left\{\underline{0}_{(N-1)}\right\} \subset \mathbb{R}^{N-1},
$$

we see that by construction

$$
\Omega \backslash\left\{\underline{0}_{(N-1)}\right\} \subseteq \underline{\Omega} \subseteq \underline{\Omega}^{\prime} .
$$

Consequently, if Algorithm 1 provides a feasible result, then all conditions of Proposition 11 and, hence, also of Theorem 12 are satisfied. In case the numerical evaluation was inconclusive, the droop parameters $K$ and virtual inertias $M$ could be modified to ease feasibility of the proposed synchronization conditions. To this end, Lemma 14 and the discussion following it provide some guidelines.

By applying this numerical evaluation method to our test MG we find that the conditions in Assumption 10 are verified for $\left|\eta^{*}\right| \leq 18^{\circ}$. Hence-as was to be expected due to the employed bounds in the derivation of (47) the resulting admissible region of stationary angles $\eta^{*}$ is slightly smaller than that obtained via the graphical evaluation procedure in Section 4.1. Yet it has the advantage of being easily applicable also for MGs with $N>3$.

Remark 15 The nonzero eigenvalues of $\mathcal{B}^{\top} \mathcal{B} \in$ $\mathbb{R}^{(N-1) \times(N-1)}$ are identical to those of the Laplacian matrix $\mathcal{B B}^{\top} \in \mathbb{R}^{N \times N}$ associated with the unweighted graph describing the power network topology. Hence, since the power network is assumed to be connected, $\lambda_{\min }\left(\mathcal{B}^{\top} \mathcal{B}\right)>0$ denotes the algebraic connectivitiy, i.e., the second smallest eigenvalue of the graph Laplacian $\mathcal{B B}^{\top}$, and $\lambda_{\max }\left(\mathcal{B}^{\top} \mathcal{B}\right)$ its largest eigenvalue. However, these terms only appear in (44) as a consequence of the employed bounds and therefore may not offer a direct physical interpretation, yet they explain the assumption of a radial network topology.

\section{Conclusions and future research}

We have performed a global synchronization analysis of a radial MG model with the typical droop controlthe global qualifier being the central feature that distinguishes our results from the ones reported in the literature. The analysis consists of three main steps. First, the existence of the equilibrium sets of interest is established (Proposition 4). Second, the (local) stability properties of these equilibrium sets are characterized (Lemma 7). Third, sufficient conditions for global boundedness of solutions (Proposition 11) are given and it is shown that these conditions also imply almost global synchronization (Theorem 12). With the help of Lemma 14, a physical interpretation in terms of the droop and (virtual) inertia coefficients as well as the "degree of interconnection" of the network is provided. Finally it is shown how the proposed conditions can be verified both graphically and numerically.

The proofs of the main results are established by using the multivariable cell structure approach together with the concept of Leonov functions for periodic systems with multiple invariant sets, both of which were recently introduced in [46,1]. The main advantage of using Leonov, instead of the usual Lyapunov, functions is that the conditions of sign definiteness imposed for the latter are obviated. This key feature turns out to be essential for the analysis of MGs carried out in this paper.

Our current and future research is geared towards the extension of the present analysis to more general network 
topologies as well as more detailed models of closed-loop inverter and generator dynamics, which are described here by the swing equation. We also intend to extend the presented results to a robust stability analysis with respect to external perturbations, such as load variations, via the ISS approach derived in [56]. Another objective is to extend the employed framework to Control Leonov Functions (CLeFs) and use these to design globally stabilizing inverter controllers for MG applications.

\section{References}

[1] D. Efimov, J. Schiffer, On boundedness of solutions of periodic systems: a multivariable cell structure approach, IEEE Transactions on Automatic Control (in press) (2019).

[2] W. Winter, K. Elkington, G. Bareux, J. Kostevc, Pushing the limits: Europe's new grid: Innovative tools to combat transmission bottlenecks and reduced inertia, IEEE Power and Energy Magazine 13 (1) (2015) 60-74 (2015).

[3] F. Milano, F. Dörfler, G. Hug, D. J. Hill, G. Verbič, Foundations and challenges of low-inertia systems, in: 2018 Power Systems Computation Conference (PSCC), IEEE, 2018, pp. 1-25 (2018).

[4] N. Hatziargyriou, H. Asano, R. Iravani, C. Marnay, Microgrids, IEEE Power and Energy Magazine 5 (4) (2007) 78-94 (2007).

[5] F. Katiraei, R. Iravani, N. Hatziargyriou, A. Dimeas, Microgrids management, IEEE Power and Energy Magazine 6 (3) (2008) 54-65 (2008).

[6] G. Strbac, N. Hatziargyriou, J. P. Lopes, C. Moreira, A. Dimeas, D. Papadaskalopoulos, Microgrids: Enhancing the resilience of the European Megagrid, IEEE Power and Energy Magazine 13 (3) (2015) 35-43 (2015).

[7] J. Schiffer, D. Zonetti, R. Ortega, A. M. Stanković, T. Sezi, J. Raisch, A survey on modeling of microgrids from fundamental physics to phasors and voltage sources, Automatica 74 (2016) 135-150 (2016).

[8] J. Guerrero, J. Vasquez, J. Matas, L. de Vicuna, M. Castilla, Hierarchical control of droop-controlled AC and DC microgrids; a general approach toward standardization, IEEE Transactions on Industrial Electronics 58 (1) (2011) $158-172$ (jan. 2011).

[9] J. Rocabert, A. Luna, F. Blaabjerg, P. Rodriguez, Control of power converters in AC microgrids, IEEE Transactions on Power Electronics 27 (11) (2012) 4734-4749 (Nov. 2012).

[10] J. Guerrero, P. Loh, M. Chandorkar, T. Lee, Advanced control architectures for intelligent microgrids - part I: Decentralized and hierarchical control, IEEE Transactions on Industrial Electronics 60 (4) (2013) 1254-1262 (2013).

[11] M. Chandorkar, D. Divan, R. Adapa, Control of parallel connected inverters in standalone AC supply systems.

[12] Q. Zhong, G. Weiss, Synchronverters: Inverters that mimic synchronous generators, IEEE Transactions on Industrial Electronics 58 (4) (2011) 1259 -1267 (april 2011).

[13] S. D'Arco, J. A. Suul, Virtual synchronous machinesclassification of implementations and analysis of equivalence to droop controllers for microgrids, in: PowerTech, 2013 IEEE Grenoble, IEEE, 2013, pp. 1-7 (2013).

[14] C. Arghir, T. Jouini, F. Dörfler, Grid-forming control for power converters based on matching of synchronous machines, Automatica 95 (2018) 273-282 (2018).
[15] B. B. Johnson, M. Sinha, N. G. Ainsworth, F. Dörfler, S. V. Dhople, Synthesizing virtual oscillators to control islanded inverters, IEEE Transactions on Power Electronics 31 (8) (2016) 6002-6015 (2016).

[16] A. H. Etemadi, E. J. Davison, R. Iravani, A decentralized robust control strategy for multi-der microgrids-Part I: fundamental concepts, IEEE Transactions on Power Delivery 27 (4) (2012) 1843-1853 (2012).

[17] S. Riverso, F. Sarzo, G. Ferrari-Trecate, et al., Plug-andplay voltage and frequency control of islanded microgrids with meshed topology., IEEE Transactions Smart Grid 6 (3) (2015) 1176-1184 (2015).

[18] M. Tucci, G. Ferrari-Trecate, Voltage and frequency control in AC islanded microgrids: a scalable, line-independent design algorithm, IFAC-PapersOnLine 50 (1) (2017) 13922-13927 (2017).

[19] M. Cucuzzella, G. P. Incremona, A. Ferrara, Decentralized sliding mode control of islanded AC microgrids with arbitrary topology, IEEE Transactions on Industrial Electronics 64 (8) (2017) 6706-6713 (2017).

[20] J. Schiffer, R. Ortega, A. Astolfi, J. Raisch, T. Sezi, Conditions for stability of droop-controlled inverter-based microgrids, Automatica 50 (10) (2014) 2457-2469 (2014).

[21] J. Schiffer, D. Goldin, J. Raisch, T. Sezi, Synchronization of droop-controlled microgrids with distributed rotational and electronic generation, in: 52nd IEEE Conference on Decision and Control, Florence, Italy, 2013, pp. 2334-2339 (2013).

[22] D. Efimov, R. Ortega, J. Schiffer, ISS of multistable systems with delays: application to droop-controlled inverter-based microgrids, in: American Control Conference, Chicago, IL, USA, 2015, pp. 4664-4669 (2015).

[23] J. Schiffer, E. Fridman, R. Ortega, J. Raisch, Stability of a class of delayed port-hamiltonian systems with application to microgrids with distributed rotational and electronic generation, Automatica 74 (2016) 71-79 (2016).

[24] J. Schiffer, C. A. Hans, T. Kral, R. Ortega, J. Raisch, Modeling, analysis, and experimental validation of clock drift effects in low-inertia power systems, IEEE Transactions on Industrial Electronics 64 (7) (2017) 5942-5951 (2017).

[25] M. Colombino, D. Groß, F. Dörfler, Global phase and voltage synchronization for power inverters: a decentralized consensus-inspired approach, in: IEEE 56th Conference on Decision and Control, 2017, pp. 5690-5695 (2017).

[26] J.-S. Brouillon, M. Colombino, D. Groß, F. Dörfler, The effect of transmission-line dynamics on a globally synchronizing controller for power inverters, in: European Control Conference, 2018, pp. 2242-2247 (2018).

[27] W. H. Kersting, Distribution system modeling and analysis, CRC press, 2012 (2012).

[28] K. Strunz, S. Barsali, Z. Styczynski, CIGRE Task Force C6. 04.02: Developing benchmark models for integrating distributed energy resources, in: Proceedings of the CIGRE 5th Southern Africa regional conference: study committee C6 colloquium, 2005 (2005).

[29] K. Rudion, A. Orths, Z. Styczynski, K. Strunz, Design of benchmark of medium voltage distribution network for investigation of DG integration, in: IEEE PESGM, 2006 (2006).

[30] J. W. Simpson-Porco, F. Dörfler, F. Bullo, Synchronization and power sharing for droop-controlled inverters in islanded microgrids, Automatica 49 (9) (2013) 2603-2611 (2013).

[31] C. De Persis, N. Monshizadeh, Bregman storage functions for microgrid control, IEEE Transactions on Automatic Control 63 (1) (2018) 53-68 (2018). 
[32] Z. Nitecki, M. Shub, Filtrations, decompositions, and explosions, American Journal of Mathematics 97 (4) (1975) 1029-1047 (1975).

[33] A. Gelig, G. Leonov, V. Yakubovich, Stability of nonlinear systems with nonunique equilibrium position, Izdatel'stvo Nauka, 1978 (1978).

[34] A. Rantzer, A dual to Lyapunov's stability theorem, Systems \& Control Letters 42 (3) (2001) 161-168 (2001).

[35] D. Angeli, J. E. Ferrell, E. D. Sontag, Detection of multistability, bifurcations, and hysteresis in a large class of biological positive-feedback systems, Proceedings of the National Academy of Sciences 101 (7) (2004) 1822-1827 (2004).

[36] G.-B. Stan, R. Sepulchre, Analysis of interconnected oscillators by dissipativity theory, IEEE Transactions on Automatic Control 52 (2) (2007) 256-270 (2007).

[37] D. V. Efimov, A. L. Fradkov, Oscillatority of nonlinear systems with static feedback, SIAM Journal on Control and Optimization 48 (2) (2009) 618-640 (2009).

[38] D. Efimov, Global Lyapunov analysis of multistable nonlinear systems, SIAM Journal on Control and Optimization 50 (5) (2012) 3132-3154 (2012).

[39] D. Angeli, D. Efimov, Characterizations of input-to-state stability for systems with multiple invariant sets, IEEE Transactions on Automatic Control 60 (12) (2015) 3242-3256 (2015)

[40] P. Forni, D. Angeli, Characterizations of integral input-tostate stability for systems with multiple invariant sets, IEEE Transactions on Automatic Control 62 (8) (2017) 3729-3743 (2017).

[41] G. Leonov, On the boundedness of the trajectories of phase systems, Siberian Mathematical Journal 15 (3) (1974) 491495 (1974).

[42] G. A. Leonov, Phase synchronization: Theory and applications, Automation and Remote Control 67 (10) (2006) 1573-1609 (2006).

[43] E. J. Noldus, New direct Lyapunov-type method for studying synchronization problems, Automatica 13 (2) (1977) 139-151 (1977).

[44] N. Barabanov, J. Schiffer, R. Ortega, D. Efimov, Conditions for almost global attractivity of a synchronous generator connected to an infinite bus, IEEE Transactions on Automatic Control 62 (10) (2017) 4905-4916 (Oct 2017).

[45] J. Schiffer, D. Efimov, R. Ortega, N. Barabanov, An inputto-state stability approach to verify almost global stability of a synchronous-machine- infinite-bus system, Philosophical Transactions of the Royal Society of London. A (1887-1895) (Aug. 2017).

[46] D. Efimov, J. Schiffer, A new criterion for boundedness of solutions for a class of periodic systems, in: European Control Conference, 2018, pp. 1642-1647 (2018).

[47] H. K. Khalil, Nonlinear systems, Vol. 3, Prentice Hall, 2002 (2002).

[48] A. van der Schaft, L2-gain and passivity techniques in nonlinear control, Springer, 2000 (2000).

[49] F. Dörfler, F. Bullo, Synchronization in complex networks of phase oscillators: A survey, Automatica 50 (6) (2014) 15391564 (2014)

[50] F. A. Rodrigues, T. K. D. Peron, P. Ji, J. Kurths, The Kuramoto model in complex networks, Physics Reports 610 (2016) 1-98 (2016)
[51] Y. Tang, F. Qian, H. Gao, J. Kurths, Synchronization in complex networks and its application-a survey of recent advances and challenges, Annual Reviews in Control 38 (2) (2014) 184-198 (2014).

[52] J. Schiffer, D. Efimov, R. Ortega, Almost global synchronization in radial multi-machine power systems, in: 57th IEEE Conference on Decision and Control (CDC), 2018 (2018)

[53] H.-D. Chiang, F. Wu, P. Varaiya, A BCU method for direct analysis of power system transient stability, IEEE Transactions on Power Systems 9 (3) (1994) 1194 -1208 (aug 1994).

[54] T. L. Vu, K. Turitsyn, Lyapunov functions family approach to transient stability assessment, IEEE Transactions on Power Systems 31 (2) (2016) 1269-1277 (2016).

[55] P. Kundur, Power system stability and control, McGraw-Hill, 1994 (1994).

[56] D. Efimov, J. Schiffer, N. Barabanov, R. Ortega, A relaxed characterization of ISS for periodic systems with multiple invariant sets, European Journal of Control 37 (2017) 1-7 (2017)

[57] M. Arcak, Passivity as a design tool for group coordination, IEEE Transactions on Automatic Control 52 (8) (2007) 13801390 (2007).

[58] S. Trip, M. Bürger, C. De Persis, An internal model approach to (optimal) frequency regulation in power grids with timevarying voltages, Automatica 64 (2016) 240-253 (2016).

[59] D. Manik, M. Timme, D. Witthaut, Cycle flows and multistability in oscillatory networks, Chaos: An Interdisciplinary Journal of Nonlinear Science 27 (8) (2017) 083123 (2017).

[60] D. Carlson, H. Schneider, Inertia theorems for matrices: The semidefinite case, Journal of Mathematical Analysis and Applications 6 (3) (1963) 430-446 (1963).

[61] P. Monzón, R. Potrie, Local and global aspects of almost global stability, in: 45th IEEE Conference on Decision and Control, 2006, pp. 5120-5125 (2006).

[62] J. Löfberg, YALMIP: toolbox for modeling and optimization in MATLAB, in: IEEE International Symposium on Computer Aided Control Systems Design, 2004, pp. 284-289 (sept. 2004).

\section{Appendix}

\section{A Proof of Lemma 13}

We give the proof of Lemma 13 . The fact that

$$
\nabla^{2} U\left(\tilde{\eta}+\eta^{*}\right)=\operatorname{diag}\left(a_{i} \cos \left(\tilde{\eta}_{i}+\eta_{i}^{*}\right)\right)
$$

together with the definition of $S\left(\tilde{\eta}+\eta^{*}\right)$ in (30) implies that $\rho$ in (39) is well-defined. To prove (40), we note that since $\mathcal{B} S\left(\tilde{\eta}+\eta^{*}\right)$ is symmetric we have that

$$
\left\|\mathcal{B} S\left(\tilde{\eta}+\eta^{*}\right)\right\|_{2}=\sup _{v \in \mathbb{R}^{N},|v|=1}\left|v^{\top} \mathcal{B} S\left(\tilde{\eta}+\eta^{*}\right) v\right|
$$


and that

$$
\begin{aligned}
\rho & =\sup _{\tilde{\eta} \in \mathbb{R}^{(N-1)}} \sup _{v \in \mathbb{R}^{N},|v|=1}\left|v^{\top} \mathcal{B} S\left(\tilde{\eta}+\eta^{*}\right) v\right| \\
& =\sup _{\tilde{\eta} \in \mathbb{R}^{(N-1)}} \sup _{v \in \mathbb{R}^{N},|v|=1}\left|v^{\top} \mathcal{B} \operatorname{diag}\left(a_{i} \cos \left(\tilde{\eta}_{i}+\eta_{i}^{*}\right)\right) \mathcal{B}^{\top} v\right|
\end{aligned}
$$

Furthermore, since the suprema in $\tilde{\eta}$ and $v$ are independent of each other, we obtain

$$
\begin{aligned}
\rho & =\sup _{v \in \mathbb{R}^{N},|v|=1}\left|v^{\top} \mathcal{B}\left[\sup _{\tilde{\eta} \in \mathbb{R}^{(N-1)}} \operatorname{diag}\left(a_{i} \cos \left(\tilde{\eta}_{i}+\eta_{i}^{*}\right)\right)\right] \mathcal{B}^{\top} v\right| \\
& =\sup _{v \in \mathbb{R}^{N},|v|=1}\left|v^{\top} \mathcal{B}\left[\operatorname{diag}\left(a_{i} \cos (0)\right)\right] \mathcal{B}^{\top} v\right| \\
& =\lambda_{\max }\left(\mathcal{B}\left[\operatorname{diag}\left(a_{i}\right)\right] \mathcal{B}^{\top}\right)=\lambda_{\max }\left(\mathcal{B} A \mathcal{B}^{\top}\right) .
\end{aligned}
$$

This completes the proof.

\section{B Proof of Lemma 14}

Set $\Phi=\phi I_{N}, \phi>0, \mu=0$ and $\alpha=1$. Then, $Q(\tilde{\eta})$ in (27) becomes

$Q(\tilde{\eta})=\left[\begin{array}{cc}\left(2 \frac{\phi}{k m}-\beta\right) I_{N} & -\phi \mathcal{B} S\left(\tilde{\eta}+\eta^{*}\right)+\frac{\beta}{k} I_{N} \\ \left(-\phi \mathcal{B} S\left(\tilde{\eta}+\eta^{*}\right)\right)^{\top}+\frac{\beta}{k} I_{N} & \left(\frac{2}{k}-\nu-\frac{\beta}{k^{2}}\right) I_{N}\end{array}\right]$.

The lower block of $Q(\tilde{\eta})$ is positive definite if and only if

$$
\frac{2}{k}-\nu-\frac{\beta}{k^{2}}>0
$$

with the left hand-side attaining a maximum for

$$
-\frac{2}{k^{2}}+\frac{2 k \beta}{k^{4}}=\frac{-2 k^{2}+2 k \beta}{k^{4}}=0,
$$

which yields $k^{*}=\beta$. Then (B.2) becomes

$$
\frac{2}{\beta}-\nu-\frac{1}{\beta}=\frac{1-\nu \beta}{\beta}>0,
$$

which, since $m, \rho$ and $\beta=k$ are positive parameters, is satisfied due to condition (41).

By inserting $k=k^{*}$ in (B.1) and taking the Schur com- plement, we see that $Q(\tilde{\eta})>0$ if and only if

$$
\begin{aligned}
& \frac{1-\nu \beta}{\beta}\left(\frac{2 \phi}{\beta m}-\beta\right) I_{N} \\
& -\left(\phi \mathcal{B} S\left(\tilde{\eta}+\eta^{*}\right)+I_{N}\right)\left(\left(-\phi \mathcal{B} S\left(\tilde{\eta}+\eta^{*}\right)\right)^{\top}+I_{N}\right) \\
= & \frac{1-\nu \beta}{\beta}\left(\frac{2 \phi}{\beta m}-\beta\right) I_{N}-\phi^{2} \mathcal{B} S\left(\tilde{\eta}+\eta^{*}\right) S^{\top}\left(\tilde{\eta}+\eta^{*}\right) \mathcal{B}^{\top} \\
& +\phi \mathcal{B} S\left(\tilde{\eta}+\eta^{*}\right)+\phi S^{\top}\left(\tilde{\eta}+\eta^{*}\right) \mathcal{B}^{\top}-I_{N}>0 .
\end{aligned}
$$

By using $\rho$ defined in (39), we obtain that the above condition is satisfied if

$$
\frac{1-\nu \beta}{\beta}\left(\frac{2 \phi}{\beta m}-\beta\right)-\phi^{2} \rho^{2}-2 \rho \phi-1>0,
$$

the left hand-side of which attains a maximum with respect to $\phi$ for

$$
\phi^{*}=\frac{1-\nu \beta-m \rho \beta^{2}}{\beta^{2} m \rho^{2}} .
$$

Since $m, \rho$ and $\beta=k$ are positive parameters, condition (41) implies that $\phi>0$. Furthermore, with $\phi=\phi^{*}$ the inequality (B.3) becomes

$$
\frac{(\beta \nu-1)\left(\beta^{4} m^{2} \rho^{2}+2 \beta^{2} m \rho+\nu \beta-1\right)}{\beta^{4} m^{2} \rho^{2}}>0 .
$$

Since, with $(41),(\beta \nu-1)<0$, the above inequality is equivalent to

$$
1-\nu \beta-\beta^{4} m^{2} \rho^{2}-2 \beta^{2} m \rho>0,
$$

which by inserting $\beta=k$ yields (41). The proof is completed by noting that, since we have only used strict inequalities in the above derivations, condition (41) ensures that there is a (small enough) $\mu>0$, such that $Q(\tilde{\eta})>0$ for all $\eta^{*} \in \mathbb{R}^{(N-1)}$ and all $\tilde{\eta} \in \mathbb{R}^{(N-1)}$. 